\title{
Noise at a Fermi-edge singularity in self-assembled InAs quantum dots
}

\author{
N. Maire, ${ }^{1, *}$ F. Hohls, ${ }^{1}$ T. Lüdtke, ${ }^{1}$ K. Pierz, ${ }^{2}$ and R. J. Haug ${ }^{1}$ \\ ${ }^{1}$ Institut für Festkörperphysik, Universität Hannover, Appelstrasse 2, D-30167 Hannover, Germany \\ ${ }^{2}$ Physikalisch-Technische Bundesanstalt, Bundesallee 100, D-38116 Braunschweig, Germany
}

(Received 9 March 2007; published 20 June 2007)

\begin{abstract}
We present noise measurements of self-assembled InAs quantum dots at high magnetic field. In comparison to $I$ - $V$ characteristics at zero magnetic field, we notice a strong current overshoot that is due to a Fermi-edge singularity. We observe an enhanced suppression in the shot noise power simultaneous to the current overshoot that is attributed to the electron-electron interaction at the Fermi-edge singularity.
\end{abstract}

DOI: 10.1103/PhysRevB.75.233304

PACS number(s): 73.63.Kv, 72.70.+m, 73.40.Gk

The measurement of shot noise provides information that cannot be extracted from conductance measurements alone. ${ }^{1}$ It has its origin in time dependent fluctuations of the electrical current due to the discreteness of the charge. For an uncorrelated flow of electrons, the shot noise power $S$ induced by individual tunneling events is proportional to the stationary current $I$ and the absolute charge of the electrons, $S=2 e I{ }^{2}$ Interactions between the electrons, e.g., Coulomb interaction or Pauli exclusion principle, can reduce ${ }^{3,4}$ or enhance $^{5,6}$ the shot noise power in resonant tunneling devices. For tunneling through zero dimensional states, which form in the so-called quantum dots, it has been shown both theoretically and experimentally that the shot noise power $S$ is suppressed down to half its normal value, ${ }^{7-12} e I \leqslant S$ $\leqslant 2 e I$. Recently, deviations in the shot noise power from the previous values have been proposed due to certain electronelectron interaction effects, such as the Kondo effect ${ }^{13-15}$ or cotunneling. ${ }^{16}$

Motivated by these results, we present temperature dependent noise measurements of self-assembled InAs quantum dots under the influence of a high magnetic field, leading to another electron-electron interaction effect, a so-called Fermi-edge singularity effect. This effect is caused by an interaction induced rearrangement of the states in the lead at the resonance of the dot level with the emitter lead Fermi energy. This results in an increased tunneling rate. Its dominant observable feature is a strong overshoot in the current at certain values of the bias voltage. ${ }^{17-20}$ This overshoot is accompanied by a suppression of the measured shot noise. ${ }^{21}$ We examine this effect in detail and find that this suppression is stronger than expected and reveals the interaction effect between the lead and the dot.

The active part of the investigated sample consists of a GaAs-AlAs-GaAs heterostructure with a cross section of 9 $\times 9 \mu \mathrm{m}^{2} . \mathrm{N}$-doped GaAs acts as three-dimensional emitter and collector. Situated inside the AlAs are 1.8 monolayers InAs. Due to the Stranski-Krastanov growth mechanism, InAs quantum dots (QDs) are formed with a density of $\approx 220 / \mu \mathrm{m}^{2}$; only a small fraction participate in electronic transport. ${ }^{22}$ The lower and upper AlAs tunneling barriers are 4 and $6 \mathrm{~nm}$ thick, respectively. Since transmission electron microscopy images show that the QDs have a height of 2-3 nm, the effective thickness of the AlAs barrier on top of the QDs is reduced to 3-4 $\mathrm{nm}$.

The sample is inserted into a ${ }^{3} \mathrm{He}$ system. This allows us to reach temperatures down to $T=300 \mathrm{mK}$ and magnetic fields up to $15 \mathrm{~T}$. A dc bias is applied to the sample, and the current is amplified by a low noise current amplifier with a bandwidth of $20 \mathrm{kHz}$. The dc part is monitored by a voltmeter. A fast-Fourier-transform analyzer measures the noise spectra.

The $I-V$ characteristic of the sample shows distinct steps that correspond to resonant tunneling through individual quantum dots. ${ }^{22}$ At zero bias, the ground state energy of the quantum dots is well above the Fermi energy in the leads. ${ }^{10}$ Resonant tunneling only sets in when the applied bias voltage is sufficiently large to bring the quantum dots' ground state energy on par with the emitter Fermi edge. One of these steps is shown in Fig. 1 (dashed line). The electrons first tunnel through the thick barrier into the dot and leave it through the thinner barrier (see left inset). So, we expect the tunneling rate $\Theta_{C}$ out of the dot into the collector to be higher than the emitter rate $\Theta_{E}$ out of the emitter into the dot. This is confirmed by noise measurements for another device from the same wafer structure ${ }^{23}$ and by the observation of strong fluctuations in the $I-V$ characteristic stemming from

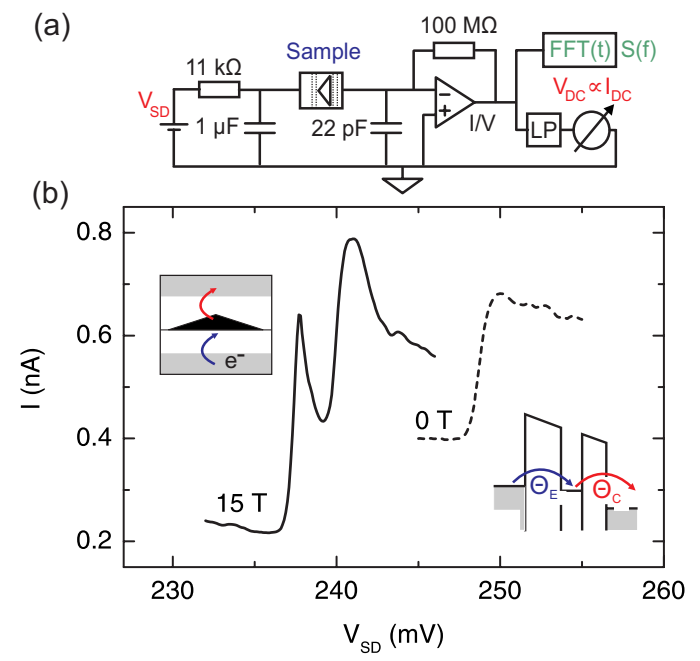

FIG. 1. (Color online) (a) Simplified equivalent circuit. (b) $I-V$ characteristic of a current step at magnetic fields of $0 \mathrm{~T}$ (dashed line) and $15 \mathrm{~T}$ (solid line) at a temperature of $0.4 \mathrm{~K}$. Left inset: Schematic of the tunneling direction through the pyramidal shaped quantum dots. Right inset: Schematic of the corresponding band diagram on resonance. 


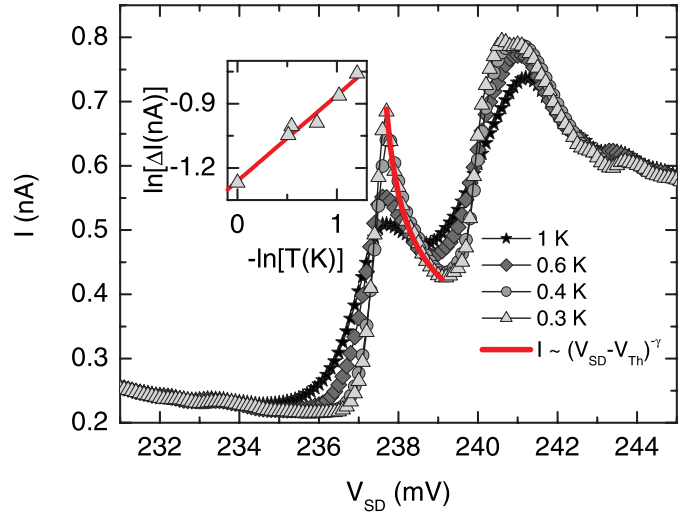

FIG. 2. (Color online) $I-V$ characteristic of the current step at $B=15 \mathrm{~T}$ for four different temperatures. Thick line: Fit using Eq. (2). Inset: Current step heights $\Delta I$ for different temperatures (symbols) and corresponding fit using Eq. (1) (line).

fluctuations of the local density of states in the emitter. ${ }^{24} \mathrm{~A}$ schematic view of the corresponding band structure is shown in the right inset.

Also shown is the same current step at a high magnetic field of $15 \mathrm{~T}$ (solid line) applied parallel to the current. The features discussed in the following were also seen down to magnetic fields of $\approx 12 \mathrm{~T} ; 15 \mathrm{~T}$ was chosen for the most distinct characteristics.

The resonance is shifted to lower bias voltages due to a redistribution of the emitter electrons into the lowest Landau level. It has split into two peaks due to the Zeeman splitting of the ground state of the quantum dot.

The more interesting feature is the strong peaklike current overshoot at the steps. The first current peak at $237.8 \mathrm{mV}$ has 1.5 times the height of the current step at $0 \mathrm{~T}$. At the second current peak, the overall current has doubled compared to $0 \mathrm{~T}$. The likely origin of this overshoot is the Fermi-edge singularity effect, which is an interaction effect between the localized electron on the dot and the electrons near the Fermi edge of the emitter. We can exclude on-dot interactions as they would lead to a decreasing current. ${ }^{25}$ One signature of the Fermi-edge singularity is that the maximum current contribution $\Delta I$ of a single spin channel follows a power law given by ${ }^{19}$

$$
\ln (\Delta I) \sim-\gamma \ln (T) .
$$

In Fig. 2, the current step at $15 \mathrm{~T}$ is shown for four different temperatures ranging from $T=0.3 \mathrm{~K}$ to $T=1 \mathrm{~K}$. The temperature dependent height of the peaks is clearly visible. In the inset in Fig. 2 a plot of $\ln (\Delta I)$ at $\approx 238 \mathrm{mV}$ vs $-\ln (T)$ is shown, $\Delta I$ being the peak current with subtracted background current. A fit using Eq. (1) yields $\gamma=0.40 \pm 0.03$. For similar measurements of a Fermi-edge singularity at InAs quantum dots, $\gamma=0.43$ was obtained. ${ }^{19}$

Another characteristic attribute of a Fermi-edge singularity effect is a voltage dependence of the current given by ${ }^{17}$

$$
I \sim\left(V_{S D}-V_{T h}\right)^{-\gamma}
$$

$V_{T h}$ corresponds to the bias voltage at which the Fermi energy of the emitter is in resonance with the ground state of

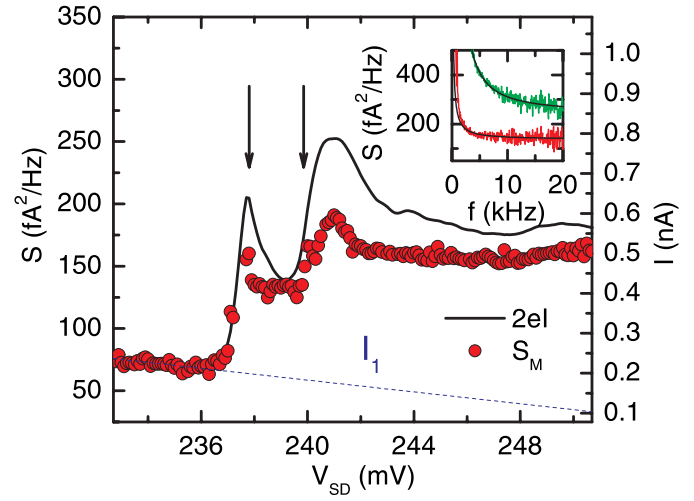

FIG. 3. (Color online) Measured shot noise power $S_{M}$ (filled dots, left axis) at $0.4 \mathrm{~K}$. The solid line corresponds to the current on the right axis and to the shot noise power of a single tunneling barrier, $S=2 e I$, on the left axis. Also shown is the linear fitted background current $I_{1}$ (dashed line). Inset: Shot noise spectra and corresponding fits at $237.8 \mathrm{mV}$ (lower spectrum) and at $239.9 \mathrm{mV}$ (upper spectrum, offset by $100 \mathrm{fA}^{2} / \mathrm{Hz}$, fit includes $\left.B / f^{\beta}\right)$.

the dot. From the $I-V$ characteristics for different temperatures, we determine $V_{T h}=237.4 \mathrm{mV}$. A fit of the $0.3 \mathrm{~K}$ data using Eq. (2) with fixed $V_{T h}$ yields $\gamma=0.47 \pm 0.01$, in fair agreement with the exponent determined above. This fit is shown by the thick line in Fig. 2 and matches the measurement well. We conclude that this current overshoot is indeed caused by a Fermi-edge singularity effect. A more sophisticated analysis of the Fermi-edge singularity effect at a current step can be found in Ref. 26.

We will now analyze the noise characteristic of this particular electron-electron interaction effect: At high frequencies, the measured noise power density is frequency independent, as expected for shot noise, while at low frequencies, an additional $1 / f$ noise appears. To remove the $1 / f$ part, a $A / f+S_{M}$ fit is carried out, $S_{M}$ being the resulting average shot noise power. For a high differential source conductance, we additionally have to account for the input voltage noise of the amplifier, which adds a term $B / f^{\beta}$, with $\beta \approx 2$. We include this term into the fit for the steep risers at $V_{S D}$ $\approx 237 \mathrm{mV}$ and $V_{S D} \approx 240 \mathrm{mV}$. $S_{M}$ is shown in Fig. 3 by the filled dots. Two spectra and their fits are shown in the inset in Fig. 3. The voltage position of the lower one corresponds to the maximum current on the Fermi-edge singularity at $237.8 \mathrm{mV}$ (left arrow in Fig. 3). The voltage position of the upper spectrum is at $239.9 \mathrm{mV}$ (right arrow) at a very high differential conductance (fit includes $B / f^{\beta}$ ). Comparing $S_{M}$ to the full shot noise $S=2 e I$ of single barrier tunneling, we find the expected suppression of shot noise on resonance.

To better characterize the degree of shot noise suppression, the Fano factor $\alpha=S_{M} / 2 e I$ is introduced. For zero temperature, the Fano factor $\alpha$ for a single weakly coupled ground state can be described by ${ }^{11,12}$

$$
\alpha=1-\frac{2 \Theta_{E} \Theta_{C}}{\left(\Theta_{E}+\Theta_{C}\right)^{2}},
$$

with the emitter-dot tunneling rate $\Theta_{E}$ and collector-dot tunneling rate $\Theta_{C}$. For a quantum dot, $\alpha$ is expected to be in the 


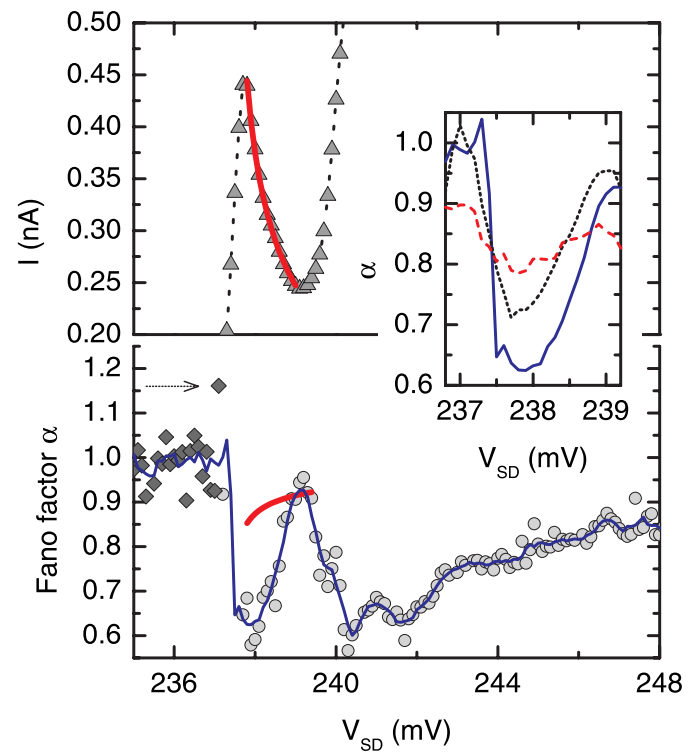

FIG. 4. (Color online) Fano factor $\alpha$ (lower panel) at $0.4 \mathrm{~K}$ using $\alpha=S_{M} / 2 e I$ (diamonds) and $\alpha_{2}$ (see text, circles). The drawn through line is a boxcar average. The thick solid line is a test of Eq. (3) (see text). Also shown is the corresponding current $I$ (upper panel) and its fit using Eq. (4) (see text, solid line). Inset: Boxcar averages of the Fano factor for $0.4 \mathrm{~K}$ (solid), $0.6 \mathrm{~K}$ (dotted), and $1 \mathrm{~K}$ (dashed line).

range of $0.5-1,0.5$ for symmetrical barriers $\left(\Theta_{E}=\Theta_{C}\right)$ and close to 1 for very asymmetric barriers.

In Fig. 4, the measured Fano factor is shown. Below $237 \mathrm{mV}, \alpha \approx 1$ is observed. This can be explained in the following way: At $\approx 220 \mathrm{mV}$, resonant tunneling through another quantum dot sets in. For a resonant transport at voltages sufficiently far away from the onset voltage, a Fano factor of $\alpha \approx 1$ is expected. ${ }^{23}$ We see an enhanced shot noise $(\alpha=1.16$, see arrow in Fig. 4) just at the beginning of the current step at $237.1 \mathrm{mV}$. The origin of this overshoot is unclear, but we observe it consistently for each measurement. With increasing temperatures, the overshoot gets less until it has completely vanished at $1 \mathrm{~K}$. Super-Poissonian noise has also been recently observed at different quantum dot systems. ${ }^{5,6}$

To extract the Fano factor originating only from the quantum dot participating in the resonant tunneling process with the Fermi-edge singularity effect, we have to subtract the influence of the other aforementioned dot with a resonance at $\approx 220 \mathrm{mV}$. The contribution $\alpha_{1,2}$ of the two quantum dots to the Fano factor is given by their fractions $I_{1,2}$ of the overall current $I \cdot{ }^{10,27}$ A linear fit of the current $I_{1}$ of the dot with a resonance at $220 \mathrm{mV}$ is depicted by the dashed line in Fig. 3. If we now calculate $\alpha_{2}$ with an $\alpha_{1}=1$ and the linear fitted current $I_{1}$, we get the Fano factor given by the circles in Fig. 4. The line is a three point boxcar average of these data.

As can be seen, the Fano factor $\alpha$ exhibits a very sharp dip at the onset of the current step $\left(V_{S D}=237 \mathrm{mV}\right)$ with a maximum suppression at the voltage position of the current peak. For a further increased voltage, we observe a strong rise in $\alpha$ parallel to a large decrease in current. The pattern is repeated when the upper Zeeman level of the quantum dot comes into resonance at $V_{S D} \approx 240 \mathrm{mV}$. We will restrict the following discussion of the Fano factor to the range $V_{S D}$ $\leqslant 239 \mathrm{mV}$, where the second Zeeman level can be safely ignored as only a very small fraction of the current is carried by this second level.

The initial drop of $\alpha$ can be qualitatively explained following Ref. 23: At the onset of resonant tunneling, only the highest energetic emitter electrons in the tail of the emitter Fermi distribution function $f_{E}$ can participate in a resonant tunneling process, and the effective tunneling rate can be written as $\Theta_{E}=\Theta_{E}^{0} f_{E}$, with $f_{E} \ll 1$, and thus $\Theta_{E} \ll \Theta_{C}$. Therefore, we start with $\alpha \approx 1$. With increasing bias voltage, the energy level of the dot is shifted downward with respect to the Fermi level of the emitter and $f_{E}=0 \rightarrow 1$. $\Theta_{E}$ increases accordingly, leading to a rise in the current and, with Eq. (3), to a decrease in the Fano factor.

In our previous measurements of similar devices at $B=0$, we have shown that the initial drop of the Fano factor is followed by a gentle rise over several tens of $\mathrm{mV}$, which mirrors the density of states in the emitter. ${ }^{23}$ In our measurement here for $15 \mathrm{~T}$, we observe a change of $\alpha$ on a totally different voltage scale: The Fano factor rises rapidly from $\alpha=0.58$ at $237.9 \mathrm{mV}$ to $\alpha=0.91$ at $238.9 \mathrm{mV}$. Both Fano factor and current change dramatically within a voltage range of only $1 \mathrm{mV}$ !

We indeed anticipate a rapid change near the Fermi energy in the presence of a Fermi-edge singularity. We will now try whether the measured current $I$ and Fano factor $\alpha$ can be modeled by the introduction of an interaction enhanced emitter tunneling rate $\Theta_{E}$ into the semiclassical relations for $I$ and $\alpha$. In the zero temperature limit, $\Theta_{E}$ is predicted to follow ${ }^{28}$

$$
\Theta_{E}\left(V_{S D}\right)=\Theta_{E}^{0}\left(\frac{D}{e\left(V_{S D}-V_{T h}\right)}\right)^{\gamma}
$$

for a voltage near but not too close to the threshold voltage $V_{T h}$. Following Ref. 28, the increase of the current peak can then be described by

$$
I=e \frac{\Theta_{C} \Theta_{E}\left(V_{S D}\right)}{\Theta_{C}+\Theta_{E}\left(V_{S D}\right)} .
$$

Due to the high bias, the change of the collector tunneling rate $\Theta_{C}$ is negligible in the range of interest, ${ }^{23}$ and we determine it far away from the resonances where the effect of the Fermi-edge singularity is negligible $\left(\Theta_{C}=3.4 \times 10^{10} \mathrm{~s}^{-1}\right.$ at $V_{S D}=248 \mathrm{mV}$ ). Inserting $\Theta_{C}$ in Eq. (5) and fitting the first current peak $\left[V_{T h}=237.4 \mathrm{mV}\right.$ fixed; $\gamma=0.45$ and $\Theta_{E}^{0}(D / e)^{\gamma}$ $=8.4 \times 10^{7} \mathrm{~V}^{\gamma} / \mathrm{s}$ fitted $]$ gives a sound agreement with the measurement as depicted in Fig. 4 by the thick solid line in the upper panel.

We can now insert the above determined interaction enhanced and voltage dependent tunneling rate $\Theta_{E}\left(V_{S D}\right)$ into the semiclassical equation [Eq. (3)]. The resulting prediction for $\alpha$ is depicted by the thick solid line in the lower panel of Fig. 4. The values calculated at some distance to the Fermiedge singularity where the interaction effects are weak are in reasonable agreement with our measurement. However, near the Fermi-edge singularity, we observe a significant discrep- 
ancy: The calculated shot noise suppression falls way short of the measured strong suppression; the change of $\alpha$ is much more rapid.

Thus, we find that Eq. (3), a relation that was deduced with only an on-site Coulomb repulsion as the interaction, is not applicable near the Fermi-edge singularity. Instead, we observe a strongly reduced Fano factor hinting on additional anticorrelations of the tunneling events due to the interaction between the lead and the dot at the Fermi-edge singularity.

The strong impact of the Fermi-edge singularity on the shot noise is further confirmed by the influence of temperature (see inset in Fig. 4). We observe a much stronger temperature dependence than expected when just using the changing Fermi function $f_{E}$ in the effective tunneling rate $\Theta_{E}=\Theta_{E}^{0} f_{E}$ at the step edge. ${ }^{23}$

We conclude that the current noise in the regime of the Fermi-edge singularity reveals the interaction between the emitter and the dot. Only a theory that accounts for this interaction will be able to describe the shot noise near the singularity. The relevance of interactions between the lead and the dot was pointed out for quantum dots in the regime of large tunnel coupling where the Kondo effect is observed.
A number of theoretical papers ${ }^{13-15}$ emphasized the importance of noise measurements to probe the Kondo regime, but due to the difficulty of the measurement, experimental data on this regime are still missing. The need for a more complete consideration of interaction effects also at lower couplings was demonstrated in calculations for an Andersonimpurity model with finite spin splitting ${ }^{16}$ and now awaits experimental verification. For both the above-mentioned Kondo and cotunneling regime, the spin degree of freedom on the dot was essential. In contrast, our experiment demonstrates the relevance of interaction for a single level system with only one spin species involved. Thus, besides experimentally demonstrating the relevance of shot noise to examine the dot-lead interaction, we also reveal the importance of current correlations in a new regime.

In summary, we have measured the shot noise at a Fermiedge singularity. We have observed a strong shot noise suppression, which we attribute to the strong interaction between the lead and the dot at the Fermi-edge singularity.

We acknowledge the financial support from BMBF via the program "NanoQUIT." *maire@nano.uni-hannover.de

${ }^{1}$ Y. M. Blanter and M. Büttiker, Phys. Rep. 336, 1 (2000).

${ }^{2}$ W. Schottky, Ann. Phys. 57, 541 (1918).

${ }^{3}$ Y. P. Li, A. Zaslavsky, D. C. Tsui, M. Santos, and M. Shayegan, Phys. Rev. B 41, 8388 (1990).

${ }^{4}$ H. C. Liu, J. Li, G. C. Aers, C. R. Leavens, M. Buchanan, and Z. R. Wasilewski, Phys. Rev. B 51, 5116 (1995).

${ }^{5}$ P. Barthold, F. Hohls, N. Maire, K. Pierz, and R. J. Haug, Phys. Rev. Lett. 96, 246804 (2006).

${ }^{6}$ S. Gustavsson, R. Leturcq, B. Simovic, R. Schleser, P. Studerus, T. Ihn, K. Ensslin, D. C. Driscoll, and A. C. Gossard, Phys. Rev. B 74, 195305 (2006).

${ }^{7}$ H. Birk, M. J. M. de Jong, and C. Schönenberger, Phys. Rev. Lett. 75, 1610 (1995).

${ }^{8}$ L. Y. Chen and C. S. Ting, Phys. Rev. B 43, 4534 (1991).

${ }^{9}$ J. H. Davies, P. Hyldgaard, S. Hershfield, and J. W. Wilkins, Phys. Rev. B 46, 9620 (1992).

${ }^{10}$ A. Nauen, I. Hapke-Wurst, F. Hohls, U. Zeitler, R. J. Haug, and K. Pierz, Phys. Rev. B 66, 161303(R) (2002).

${ }^{11}$ G. Kiesslich, A. Wacker, and E. Schöll, Phys. Rev. B 68, 125320 (2003).

${ }^{12}$ A. Thielmann, M. H. Hettler, J. König, and G. Schön, Phys. Rev. B 68, 115105 (2003).

${ }^{13}$ Y. Meir and A. Golub, Phys. Rev. Lett. 88, 116802 (2002).

${ }^{14}$ R. Lopez and D. Sanchez, Phys. Rev. Lett. 90, 116602 (2003).

${ }^{15}$ E. Sela, Y. Oreg, F. von Oppen, and J. Koch, Phys. Rev. Lett. 97, 086601 (2006).
${ }^{16}$ A. Thielmann, M. H. Hettler, J. König, and G. Schön, Phys. Rev. Lett. 95, 146806 (2005).

${ }^{17}$ A. K. Geim, P. C. Main, N. La Scala, Jr., L. Eaves, T. J. Foster, P. H. Beton, J. W. Sakai, F. W. Sheard, M. Henini, G. Hill, and M. A. Pate, Phys. Rev. Lett. 72, 2061 (1994).

${ }^{18}$ K. A. Benedict, A. S. G. Thornton, T. Ihn, P. C. Main, L. Eaves, and M. Henini, Physica B 256-258, 519 (1998).

${ }^{19}$ I. Hapke-Wurst, U. Zeitler, H. Frahm, A. G. M. Jansen, R. J. Haug, and K. Pierz, Phys. Rev. B 62, 12621 (2000).

${ }^{20}$ E. E. Vdovin, Yu. N. Khanin, O. Makarovsky, Yu. V. Dubrovskii, A. Patanè, L. Eaves, M. Henini, C. J. Mellor, K. A. Benedict, and R. Airey, Phys. Rev. B 75, 115315 (2007).

${ }^{21}$ N. Maire, F. Hohls, T. Lüdtke, R. J. Haug, and K. Pierz, Physica E (Amsterdam) 34, 508 (2006).

${ }^{22}$ I. Hapke-Wurst, U. Zeitler, U. F. Keyser, R. J. Haug, K. Pierz, and Z. Ma, Appl. Phys. Lett. 82, 1209 (2003).

${ }^{23}$ A. Nauen, F. Hohls, N. Maire, K. Pierz, and R. J. Haug, Phys. Rev. B 70, 033305 (2004)

${ }^{24}$ T. Schmidt, R. J. Haug, V. I. Fal'ko, K. v. Klitzing, A. Förster, and H. Lüth, Europhys. Lett. 36, 61 (1996).

${ }^{25}$ M. Rontani and E. Molinari, Phys. Rev. B 71, 233106 (2005).

${ }^{26}$ H. Frahm, C. von Zobeltitz, N. Maire, and R. J. Haug, Phys. Rev. B 74, 035329 (2006).

${ }^{27}$ G. Kiesslich, A. Wacker, E. Schöll, A. Nauen, F. Hohls, and R. J. Haug, Phys. Status Solidi C 0, 1293 (2003).

${ }^{28}$ K. A. Matveev and A. I. Larkin, Phys. Rev. B 46, 15337 (1992). 